\title{
Boundary Integral Solutions of the Heat Equation
}

\author{
By E. A. McIntyre, Jr.*
}

\begin{abstract}
The Boundary Integral Method (BIM) has recently become quite popular because of its ability to provide cheap numerical solutions to the Laplace equation. This paper describes an attempt to apply a similar approach to the (time-dependent) heat equation in two space variables.
\end{abstract}

1. Introduction. In its simplest form, the BIM uses the prescribed initial and boundary data, together with the (known) fundamental solution of a given differential equation defined on some domain $\Omega$, to construct a second integral equation which is itself to be solved on the boundary of $\Omega$. In our case, the integral equation solution is a layered thermal potential, i.e., it represents a continuous distribution of sources and sinks along the boundary of the two-dimensional domain $\Omega$. One then obtains the solution to the given problem by integrating the product of the layered thermal potential and a known kernel over the boundary, hence the terminology boundary integral.

The advantage of such an approach is that the heart of the computation, viz. the solution of the integral equation, is performed on the boundary, thus reducing the problem from two space dimensions to one. Furthermore, the resulting domain in one space and time will be rectangular, a computational convenience, even though the given domain $\Omega$ may have been quite irregular (see Example 5 in the Appendix). Of course, as a result of these simplifications, we may expect substantial savings in computer time and storage.

The work outlined below is based on the use of single layered thermal potentials, and requires that the domain have a smooth boundary and, with some restrictions, either a Neumann or mixed boundary condition. The equation itself must be homogeneous, but we do allow inhomogeneous initial data. Though not shown here, it seems to be well within the capabilities of this approach to handle boundaries involving an arbitrary mixture of piecewise $C^{2}$ curves, with Dirichlet, Neumann, and/or mixed boundary data.

Of course, the tradeoff is that to evaluate the solution, we must do a double integral for each point at which we want to know the solution. If needed at a large number of points, the cost of generating the solution dominates, and the method becomes impractical. In many applications however, in particular in semiconductor

Received November 16, 1983; revised January 21, 1985.

1980 Mathematics Subject Classification. Primary 65N30, 65R20; Secondary 35A08, 35K05, 45L10, 45-04, 65M60.

Key words and phrases. Boundary integrals, heat equation, fundamental solutions, thermal potentials, Volterra integral equations, Galerkin's method, $B$-splines, quadrature methods.

*Work done while at Bell Laboratories. 
fabrication, one only needs to know the solution at one, or perhaps a few points, late in time. In such instances, we might expect this technique to be of some value.

As far as the author knows, this is the second time the theory has been implemented directly (see [3]), though N. Ghosh (see [9]) has presented a slightly different formulation of the basic problem and there has been some work on methods employing a Laplace transform in time (see [15]). This work is an outgrowth of that of J. Blue on boundary integral solutions of the Laplace equation (see [2]). I would like also to acknowledge many very useful discussions with $L$. Kaufman, J. McKenna, and N. Shryer.

2. The Mathematical Problem. Let $\Omega$ be an open bounded set in the plane whose boundary $\partial \Omega$ is a simple closed curve in $C^{2}$, i.e.,

$$
\partial \Omega=\{\boldsymbol{\xi}(s) \mid 0 \leqslant s \leqslant L\},
$$

where $s$ is arclength, $\xi(s) \in C^{2}[0, L]$ and $\xi\left(s_{1}\right)=\xi\left(s_{2}\right)$ implies $s_{1}=s_{2}$, with the exception that $\xi(0)=\xi(L)$. Further, we assume $\beta(s, t), g(s, t) \in C(S)$, where

$$
S=\partial \Omega \times(0, T),
$$

$T<\infty$ (see Diagram 2.1). Problem: Find $u(\mathbf{x}, t)$ such that

$$
\begin{aligned}
& \text { (i) } L u=u_{t}-\Delta u=0 \text { on } \Omega \times(0, T), \\
& \text { (ii) } \frac{\partial u}{\partial \nu}+\beta(s, t) u(s, t)=g(s, t) \text { on } S,
\end{aligned}
$$

where $\partial u / \partial \nu=\nabla u \cdot \hat{\nu}_{s}, \hat{\nu}_{s}$ being the inward normal to $\partial \Omega$ at $\xi(s)$, and

$$
\text { (iii) } u(\mathbf{x}, 0)=f(\mathbf{x}) \text { on } \Omega \text {. }
$$

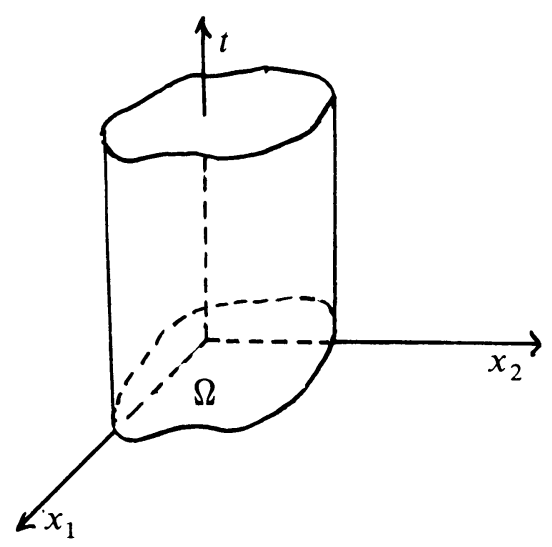

\section{Diagram 2.1}

Indeed, this is the familiar heat equation in two space variables, though, for reasons made apparent later, we have presented it under slightly more restrictive continuity assumptions than those necessary to ensure that the problem be well-posed.

A common approach to the theory of this equation involves the use of its fundamental solution to construct layered thermal potentials (see [8], [12], and [14]). We proceed in that fashion. 
For now, assume $f(\mathbf{x}) \equiv 0$. Let $\Gamma(\mathbf{x}, t ; \mathbf{y}, \tau)$ be the fundamental solution of the heat equation, i.e., for $\mathbf{x}, \mathbf{y} \in \Omega$,

$$
\Gamma(\mathbf{x}, t ; \mathbf{y}, \tau)= \begin{cases}\frac{e^{-\|\mathbf{x}-\mathbf{y}\|^{2} / 4(t-\tau)}}{4 \pi(t-\tau)}, & t>\tau, \\ 0 & \text { otherwise, }\end{cases}
$$

where $\|\cdot\|$ stands for the Euclidean norm. It can be shown (see [8]) that

$$
U(\mathbf{x}, t)=\int_{0}^{t} \int_{\partial \Omega} \Gamma(\mathbf{x}, t ; \boldsymbol{\xi}(s), \tau) \phi(s, \tau) d s d \tau,
$$

the single layered thermal potential with density $\phi$, satisfies (2.1)-(2.2) whenever $\phi$ satisfies

$$
\begin{aligned}
\frac{1}{2} \phi(s, t)= & \int_{0}^{t} \int_{\partial \Omega}\left[\frac{\partial \Gamma}{\partial \nu}(\xi(s), t ; \xi(\sigma), \tau)\right. \\
& +\beta(s, t) \Gamma(\xi(s), t ; \xi(\sigma), \tau)] \phi(\sigma, \tau) d \sigma d \tau \\
& -g(s, t),
\end{aligned}
$$

a result which derives from the jump condition

$$
\lim _{\substack{\mathbf{x} \rightarrow \boldsymbol{\xi}(s) \\(\mathbf{x} \in \Omega)}} \frac{\partial U}{\partial \nu}(\mathbf{x}, t)=-\frac{1}{2} \phi(s, t)+\frac{\partial U}{\partial \nu_{s}}(\boldsymbol{\xi}(s), t),
$$

where

$$
\frac{\partial U}{\partial \nu}(\mathbf{x}, t)=\left(\frac{\partial U}{\partial x_{1}}(\mathbf{x}, t), \frac{\partial U}{\partial x_{2}}(\mathbf{x}, t)\right) \cdot \hat{\nu}_{s} .
$$

Here, of course, $U(\mathbf{x}, 0) \equiv 0$.

Our plan then is to solve (2.4) for $\phi$, the solution $U$ being recoverable by the double integral in (2.3). Observe that, as mentioned in the preface, $\phi$ is defined on a rectangle in $s$ and $t$, which means that our problem has been reduced from two space dimensions to one.

It is of course also a feature of this method that we can solve the exterior problem too, i.e., given prescribed boundary data we can use (2.3) to solve for values of $U$ outside the region $\Omega$ (see [14]) by a change of sign in (2.5). More precisely, since $U$ is itself continuous up to the boundary, we can rewrite (2.4) as

$$
\frac{\partial U^{+}}{\partial \nu}=-\frac{\phi(s, t)}{2}+\frac{\partial U^{*}}{\partial \nu}
$$

for the interior problem, where $\partial U^{+} / \partial \nu$ represents the limit function for $\mathbf{x} \in \Omega$ and $\partial U^{*} / \partial \nu$ the first term of the double integral in (2.4), which itself derives from the formal differentiation of (2.3). Everything carries over for the exterior problem except that (2.6) becomes

$$
\frac{\partial U^{-}}{\partial \nu}=+\frac{\phi(s, t)}{2}+\frac{\partial U^{*}}{\partial \nu}
$$

where now $\partial U^{-} / \partial \nu$ represents the limit for values of $\mathbf{x}$ outside of $\bar{\Omega}$. For our purposes, we need only consider the interior problem.

Consider now the case of inhomogeneous initial data, i.e., $f(\mathbf{x})$ is not identically zero. 
It can be shown that the function

$$
v(\mathbf{x}, t)=\int_{\Omega} \frac{e^{-\|\mathbf{x}-\mathbf{y}\|^{2} / 4 t}}{4 \pi t} f(\mathbf{y}) d \mathbf{y}
$$

satisfies $L v=0$ and $\lim _{t \rightarrow 0+} v(\mathbf{x}, t)=f(\mathbf{x})$. Thus we may hope to solve the complete problem by using $v$ and the superposition of solutions to construct a modified problem for $U$. It follows then, that if $u=v+U$, where $U$ is some as yet undetermined thermal potential, we have

$$
L u=0 \text { and } \lim _{t \rightarrow 0+} u(\mathbf{x}, t)=f(\mathbf{x}) .
$$

Since we want $\partial u / \partial \nu+\beta u=g$, and we already know $v(\mathbf{x}, t)$ explicitly, we will have solved the complete problem if we can find $U$ satisfying

$$
\frac{\partial U}{\partial \nu}+\beta U=g-\frac{\partial v}{\partial \nu}-\beta v
$$

Thus, we have reduced the inhomogeneous initial data problem to a homogeneous one, and may proceed as before.

As a technical detail, we note that, on the boundary,

$$
\frac{\partial v}{\partial \nu}(s, t) \sim \frac{\partial f}{\partial \nu}+\frac{f(s)}{2\left\|\xi^{\prime}(s)\right\| \sqrt{\pi t}},
$$

as $t \rightarrow 0+$, i.e., for $f \neq 0$ on $\partial \Omega$, we have a square root singularity in $\phi$ near $t=0$. To remove this problem, we institute a change of variables, i.e., we introduce a new variable of integration $\eta^{2}=\tau$, and instead of $t$, use $\tau=\sqrt{t}$ (we apologize for the ambiguous reappearance of $\tau$ ), by means of which we may define the new functions

$$
\begin{aligned}
& \zeta(s, \tau)=\tau \phi\left(s, \tau^{2}\right)=\sqrt{t} \phi(s, t) \\
& a(s, \sigma)=\frac{(\xi(\sigma)-\xi(s)) \cdot \hat{\nu}_{s}}{4 \pi}, \\
& b(s, \tau)=\frac{\beta\left(s, \tau^{2}\right)}{2 \pi} \\
& k(s, \tau)=-2 \tau g\left(s, \tau^{2}\right)
\end{aligned}
$$

and rewrite (2.4) as

$$
\begin{aligned}
\zeta(s, \tau)=k(s, \tau)+2 \tau \int_{0}^{\tau} \int_{0}^{L} e^{-\|\xi(s)-\xi(\sigma)\|^{2} / 4\left(\tau^{2}-\eta^{2}\right)} \\
\cdot\left[\frac{a(s, \tau)}{\left(\tau^{2}-\eta^{2}\right)^{2}}+\frac{b(s, \tau)}{\left(\tau^{2}-\eta^{2}\right)}\right] \zeta(\sigma, \eta) d \sigma d \eta .
\end{aligned}
$$

This is the equation we want to solve. It is a Volterra integral equation of the second kind.

As a last consideration before turning our attention to numerical matters, we point out that the above equation has a weakly singular kernel, so that the integration in $\eta$ must take into account a square root singularity at the upper 
endpoint. More precisely, we make the following claim:

$$
\begin{gathered}
\lim _{\eta \rightarrow \tau-} \sqrt{\tau^{2}-\eta^{2}} \int_{0}^{L} e^{-\|\boldsymbol{\xi}(s)-\xi(\sigma)\|^{2} / 4\left(\tau^{2}-\eta^{2}\right)}\left[\frac{a(s, \sigma)}{\left(\tau^{2}-\eta^{2}\right)^{2}}+\frac{b(s, \tau)}{\tau^{2}-\eta^{2}}\right] \zeta(\sigma, \eta) d \sigma \\
=\frac{1}{2\left\|\boldsymbol{\xi}^{\prime}(s)\right\| \sqrt{\pi}}\left\{\frac{1}{\left\|\boldsymbol{\xi}^{\prime}(s)\right\|^{2}} \cdot \boldsymbol{\xi}^{\prime \prime}(s) \cdot \hat{\boldsymbol{\nu}}_{s}+2 \beta\left(s, \tau^{2}\right)\right\} \zeta(s, \tau) .
\end{gathered}
$$

Proof of (2.9). Clearly, for $\|\boldsymbol{\xi}(s)-\boldsymbol{\xi}(\boldsymbol{\sigma})\|$ bounded away from zero, the exponential decreases rapidly as $\eta \rightarrow \tau-$, dominating all other terms, and thus making no contribution to the desired limit. It follows then, that we need only examine effects for $\sigma$ near $s$, toward which end we consider

$$
\begin{aligned}
& C_{\delta-}=\sqrt{\tau^{2}-\eta^{2}} \int_{s-\delta}^{s} e^{-\|\xi(s)-\xi(\sigma)\|^{2} / 4\left(\tau^{2}-\eta^{2}\right)} \\
& \cdot\left[\frac{a(s, \sigma)}{\left(\tau^{2}-\eta^{2}\right)^{2}}+\frac{b(s, \tau)}{\tau^{2}-\eta^{2}}\right] \zeta(\sigma, \eta) d \sigma,
\end{aligned}
$$

where $\delta$ is a small positive constant.

If, however, we are willing to assume that $\xi(s)$ has continuous second derivatives and Taylor approximation

$$
\boldsymbol{\xi}(\sigma)=\xi(s)+\xi^{\prime}(s)(\sigma-s)+\frac{\xi^{\prime \prime}(s)}{2 !}(\sigma-s)^{2}+O(\sigma-s)^{3},
$$

we can write

$$
\|\boldsymbol{\xi}(s)-\boldsymbol{\xi}(\sigma)\|^{2}=\left\|\boldsymbol{\xi}^{\prime}(s)\right\|^{2}(\sigma-s)^{2}+O(\sigma-s)^{3},
$$

and also

$$
a(s, \sigma)=\left[\xi^{\prime}(s)(\sigma-s)+\frac{\xi^{\prime \prime}(s)}{2 !}(\sigma-s)^{2}+O(\sigma-s)^{3}\right] \cdot \frac{\hat{v}_{s}}{4 \pi},
$$

which leads to

$$
a(s, \sigma)=\frac{\xi^{\prime \prime}(s)}{8 \pi} \cdot \hat{\nu}_{s}(\sigma-s)^{2}+O(\sigma-s)^{3},
$$

since $\xi^{\prime}(s) \cdot \hat{v}_{s} \equiv 0$. Observe that it was in order to satisfy (2.11) that we chose to include a more restrictive continuity assumption in the original statement of the problem.

Substituting these expressions in (2.10) and making the change of variable $\alpha=(s-\sigma) / \sqrt{\tau^{2}-\eta^{2}}$, we have

$$
\begin{aligned}
C_{\delta-}=\int_{0}^{\delta / \sqrt{\tau^{2}-\eta^{2}}} e^{-\left[\left\|\xi^{\prime}(s)\right\|^{2}\left(\alpha^{2}\left(\tau^{2}-\eta^{2}\right)\right)+O\left(\alpha \sqrt{\tau^{2}-\eta^{2}}\right)^{3}\right] / 4\left(\tau^{2}-\eta^{2}\right)} \\
\quad \cdot\left[\frac{\alpha^{2}}{8 \pi} \xi^{\prime \prime}(s) \cdot \hat{\nu}_{s}+b(s, \tau)+O \frac{\left(\alpha \sqrt{\tau^{2}-\eta^{2}}\right)^{3}}{\left(\tau^{2}-\eta^{2}\right)}\right] \zeta\left(s-\alpha \sqrt{\tau^{2}-\eta^{2}}, \eta\right) d \alpha,
\end{aligned}
$$


and hence that

$$
\begin{aligned}
\lim _{\eta \rightarrow \tau-} C_{\delta-} & =\int_{0}^{\infty} e^{-\left\|\xi^{\prime}(s)\right\|^{2} \alpha^{2} / 4}\left[\frac{\alpha^{2}}{8 \pi} \xi^{\prime \prime}(s) \cdot \hat{\nu}_{s}+b(s, \tau)\right] d \alpha \cdot \zeta(s, \tau) \\
& =\frac{1}{4 \sqrt{\pi}\left\|\xi^{\prime}(s)\right\|}\left\{\frac{1}{\left\|\xi^{\prime}(s)\right\|^{2}} \xi^{\prime \prime}(s) \cdot \hat{\nu}_{s}+2 \beta\left(s, \tau^{2}\right)\right\} \zeta(s, \tau) .
\end{aligned}
$$

The desired result follows from the fact that we get the same limit for $\sigma \in[s, s+\delta]$.

We turn now to numerical considerations, though we must continue to bear in mind the existence of this singularity, and will, in addition, use the dependence on $\xi(s)$, and $\beta(s, t)$ shown above to determine its contribution.

3. The Numerical Method. In this section, we use Galerkin's method, based on a B-spline discretization along the boundary, to recast (2.8) as a Volterra integral equation of the second kind for the time-dependent vector of coefficients. We then present a quadrature method to solve that equation, taking into account the particular form of the singularity, and make some comments about stability.

Let us rewrite (2.8) as

$$
\zeta(s, \tau)=k(s, \tau)+2 \tau \int_{0}^{\tau} \int_{0}^{L} I(s, \tau ; \sigma, \eta) \zeta(\sigma, \eta) d \sigma d \eta
$$

Assuming some regular B-spline mesh (see [16]) in $s$, the splines being of order $k$, we can approximate $\zeta$ by a method of lines type expansion

$$
\zeta(s, \tau) \approx \sum_{i=1}^{N} \zeta_{i}(\tau) B_{i}(s)=\mathbf{Z}(\tau) \cdot \mathbf{B}(s),
$$

where the $\zeta_{i}(\tau)$ are the time-dependent coefficients, and $\mathbf{Z}$ and $\mathbf{B}$ the corresponding $N$-dimensional vectors. Substituting in (3.1) and using the Galerkin criteria, we get that

$$
\begin{aligned}
& \sum_{i=1}^{N} \zeta_{i}(\tau) \int_{0}^{L} B_{i}(s) B_{j}(s) d s \\
& =\int_{0}^{L} k(s, \tau) B_{j}(s) d s \\
& \quad+2 \tau \int_{0}^{\tau} \int_{0}^{L} \int_{0}^{L} I(s, \tau ; \sigma, \eta) \cdot \sum_{i=1}^{N} \zeta_{i}(\eta) B_{i}(\sigma) B_{j}(s) d \sigma d s d \eta
\end{aligned}
$$

$j=1,2, \ldots, N$, or, more compactly,

$$
B \cdot \mathbf{Z}(\tau)=\mathbf{K}(\tau)+2 \tau \int_{0}^{\tau} A(\tau, \eta) \cdot \mathbf{Z}(\eta) d \eta,
$$

where

$$
B=\left[\int_{0}^{L} B_{i}(s) B_{j}(s) d s\right], \quad \mathbf{K}(\tau)=\int_{0}^{L} k(s, \tau) \mathbf{B}(s) d s,
$$

and

$$
A(\tau, \eta)=\left[\int_{0}^{I} \int_{0}^{L} I(s, \tau ; \sigma, \eta) B_{i}(\sigma) B_{j}(s) d \sigma d s\right]
$$


We note in passing that, because of the support properties of B-splines, $B$ will be an invertible matrix of bandwidth $2 k-1$, from which it follows that

$$
\mathbf{Z}(\tau)=B^{-1} \mathbf{K}(\tau)+2 \tau B^{-1} \int_{0}^{\tau} A(\tau, \eta) \mathbf{Z}(\eta) d \eta,
$$

i.e., we have a linear Volterra integral equation of the second kind for the vector $\mathbf{Z}(\tau)$.

One class of methods for dealing with such problems are called quadrature methods (see [1]) which we motivate below.

Suppose we want to solve

$$
f(t)=g(t)+\lambda \int_{0}^{t} K(t, s) f(s) d s, \quad t \in[0, T],
$$

where $f$ is a scalar function and $\lambda$ is some known negative constant. This condition on $\lambda$ is necessary to ensure that we have a stable equation. Hereafter, when we use the notion of stability, it will be in reference to some particular numerical scheme, since it can be shown that (3.3) is indeed a stable equation.

One more or less straightforward approach to the solution of (3.4) is that of numerical quadrature, i.e., we set up some equally-spaced mesh $0=s_{0}<s_{1}<s_{2}<$ $\cdots<s_{n}=T$, and approximate the integral in (3.4) by a discrete sum, so that

$$
f_{i}=g_{i}+\lambda \sum_{j=0}^{i} w_{i} K_{i j} f_{j},
$$

for $i=0,1, \ldots n$, where $f_{i}=f\left(s_{i}\right), g_{i}=g\left(s_{i}\right), K_{i j}=K\left(s_{i}, s_{j}\right)$, and the $w_{i}$ are some suitably chosen quadrature weights. Thus, at each step, we will have a linear equation for $f_{i}$ in terms of the previously determined $f_{j}$, and we can solve step-by-step through the mesh.

It happens, however, that there are many ways to choose the $w_{i}$, and that some of these choices, while quite acceptable for simple quadrature, lead to serious error accumulation when employed in a scheme such as (3.5) (see [1] and [13] for a general discussion). An algorithm which seems to avoid such pitfalls is one based on the repeated Simpson's rule and trapezoidal rule.

In that scheme, we use weights based on a (three-point) repeated Simpson's rule whenever we have an odd number of points, i.e., when $i$ is even, and the same rule augmented by a (two-point) trapezoidal rule over the rightmost interval when the number of points is odd (see Diagram 3.1).

This scheme has been shown to be convergent and stable, even though it is unstable when the trapezoidal rule is applied on the left (see [10] and [13]).

Motivated by these results, we also use a repeated three-point rule with appendedon-the-right two-point rule to solve (3.3). The difference here is that the weights for the rightmost rule, whether it be for two or three points, will always be chosen to integrate a square root singularity. More precisely, our situation will be as in Diagram 3.2 , where the $S_{i j}$, unlike the other weights, vary with position, and satisfy

$$
\int_{a}^{\tau} \frac{f(s) d s}{\sqrt{\tau^{2}-s^{2}}} \approx S_{21} f(a)+S_{22} f(\tau)
$$



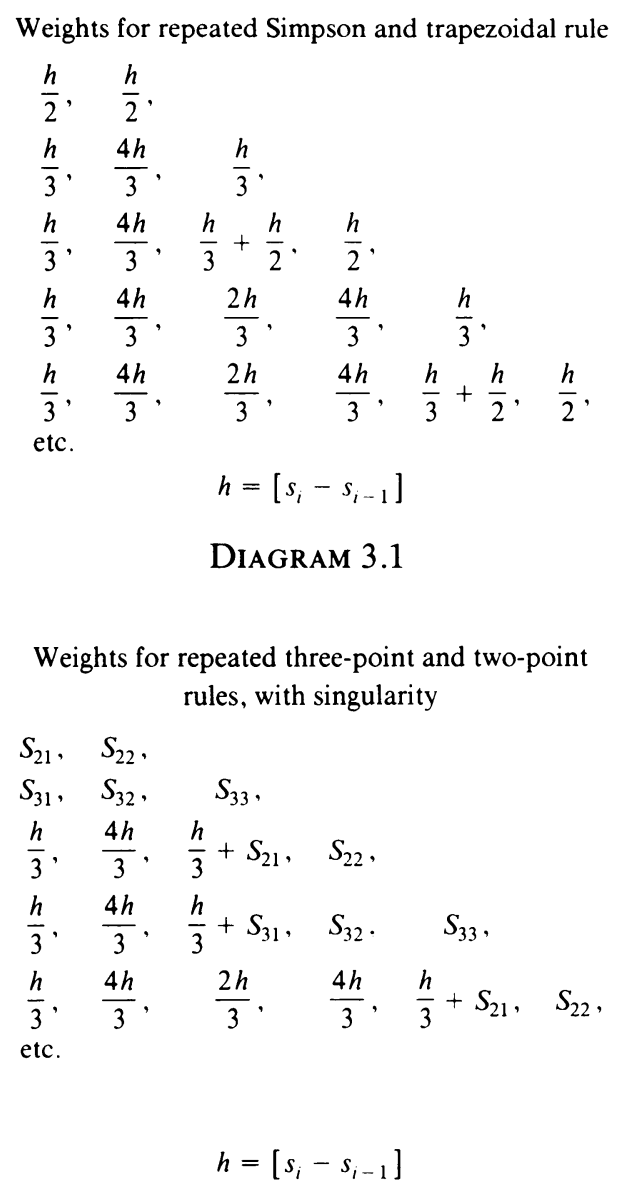

Diagram 3.2

and

$$
\int_{a}^{\tau} \frac{f(s) d s}{\sqrt{\tau^{2}-s^{2}}} \approx S_{31} f(a)+S_{32} f\left(\frac{a+\tau}{2}\right)+S_{33} f(\tau) .
$$

Our particular means of achieving (3.6)-(3.7) is the standard one, viz. that the weights be chosen so that the results are exact for polynomial functions $f$ up to first or second degree, respectively.

Of course, any choice of algorithm raises a number of theoretical issues, only a few of which we choose to address here. The few technical comments below are somewhat theoretical in nature, the material presented being in the spirit of Baker's treatment of the stability theory for Volterra equations of the second kind. Readers not interested in these issues may go directly to the examples, which can be found in an appendix in the supplements section of this issue.

Firstly, we note that the consistency of our scheme, for a large class of $\mathbf{Z}(\eta)$, follows directly from (2.9) and the error estimates for Newton-Cotes quadrature rules. Stability, on the other hand, generally requires a more complicated investigation, which to some extent explains why there remains more than one definition of it extant in the literature. 
Clearly, we can expect stability in the sense of Noble, i.e., we will not have the catastrophic growth of spurious solutions introduced by the discretization since, in keeping with his analysis, we have a repetition factor of one (see [10] and [13]). On the other hand, we do not necessarily expect that the absolute error will remain bounded-in-norm, since that condition can be shown not to hold for a simpler version of (3.4), viz. the special case $K(t, s) \equiv 1$ (see [1]). Nevertheless, one can prove block stability for small enough $h$, which leads to the conjecture that our scheme will have at most linear error growth, a situation we can indeed live with.

AT \& T Information Systems

Room 1 A308

307 Middletown-Lincroft Road

Lincroft, New Jersey 07738

1. C. T. H. BaKer, The Numerical Treatment of Integral Equations, Oxford Univ. Press, London, 1977.

2. J. L. BLUE, “Boundary integral solutions of Laplace's equation,” Bell System Tech. J., v. 57, No. 8, 1978, pp. 2797-2822.

3. Y. Chang, C. Kang \& D. Chen, "The use of fundamental Green's functions for the solution of problems of heat conduction in anisotropic media," Internat. J. Heat Mass Transfer, v. 16, 1973, pp. 1905-1918.

4. T. Cruse \& F. Rizzo, eds., Boundary-Integral Equation Method: Computational Applications in Applied Mechanics, American Society of Mechanical Engineers, 1975.

5. C. DE Boor, A Practical Guide to Splines, Springer-Verlag, Berlin and New York, 1978.

6. L. Delves \& J. Walsh, Numerical Solution of Integral Equations, Oxford Univ. Press, London, 1974.

7. P. Fox, ed., The PORT Mathematical Subroutine Library, Bell Telephone Laboratories, 1976.

8. A. Friedman, Partial Differential Equations of Parabolic Type, Prentice-Hall, Englewood Cliffs, N. J., 1964.

9. N. GHosh, On the Convergence of the Boundary Element Method, Ph. D. Thesis, Cornell University, 1982.

10. S. MCKeE \& H. BRUnNer, “The repetition factor and numerical stability of Volterra integral equations," Comput. Math. Appl., v. 6, 1980, pp. 339-347.

11. J. McKenna, private discussions.

12. S. Mikhlin, Integral Equations and Their Application to Certain Problems in Mechanics, Mathematical Physics and Technology, 2nd English ed., Macmillan, New York, 1964.

13. B. NoBLE, "Instability when solving Volterra integral equations of the second kind by multistep methods," in Conference on the Numerical Solutions of Differential Equations, Lecture Notes in Math., no. 109, Springer-Verlag, Berlin, 1969, pp. 23-39.

14. G. Polozhil, Equations of Mathematical Physics, Hayden, 1967.

15. D. SHIPPY, "Application of the boundary-integral equation method to transient phenomena in solids," in [4].

16. N. SCHRYER, A Tutorial on Galerkin's Method, using B-Splines, for Solving Differential Equations, Bell System Technical Memorandum 77-1274-1. 


\section{Supplement to \\ Boundary Integral Solutions of the Heat Equations}

By E. A. McIntyre, Jr.

Appendix: Examples

Below, we present numerical results and, where appropriate, error estimates for five examples run in single precision on the Cray-1. In all cases except the first, we have used a known solution $u$ to prescribe the boundary and initial data, and then measured the error in terms of $u$ and its computed approximation $U$. More precisely, if $\left|s_{i}\right|$ is the set of $B$-spline knots associated (implicitly) with equation (3.2), and

$$
u_{\max }(r)=\max _{1}\left|u\left(s_{1}, \tau\right)\right| .
$$

we define, for any value of $\tau$, the maximum absolute and maximum percent error as

$$
E_{\max }(\tau)-\max _{i}\left|U\left(s_{1}, \tau\right)-u\left(s_{1}, \tau\right)\right|
$$

and

$$
P_{\max }(\tau)-100 . \frac{E_{\max }(\tau)}{U_{\max }(\tau)}
$$

respectively. Our results may then be somewhat optimistic, since there remains the possibility of superconvergence at the $s_{i}$, in addition to which it may happen that large values of $u_{\max }$ generate cosmetically small $P_{\max }$.

In general, each computation, or run, consists of two calculations, or phases: the first, to compute the density function $\zeta$, and the second to "recover" the desired approximation $U$ and estimate the error

For each run, we present the number, order, and underlying mesh of the $B$-splines used to approximate $\zeta$ in equation (3.2), and the number of points used in the quadratures along the boundary. As output for phase 1, we give the condition number of the matrix $B$ in equation (3.3) and, for each time step, the maximum and minimum $B$-spline coefficients computed for the approximation to $\zeta$. We note in passing that $B$ never changes, so that we might possibly have increased our time savings by inverting it once instead of solving a linear system at each step as we have done here. 


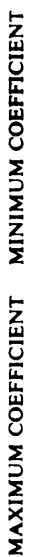

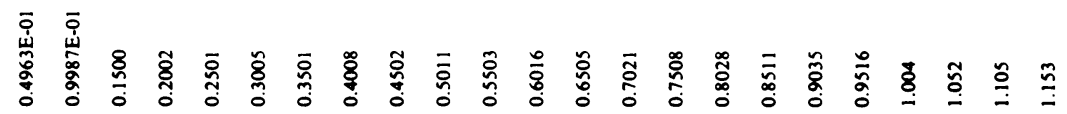

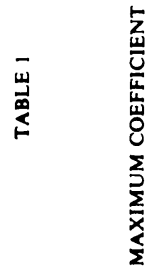

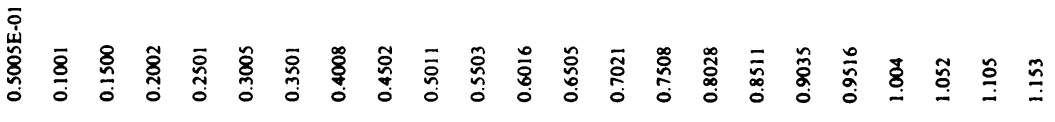

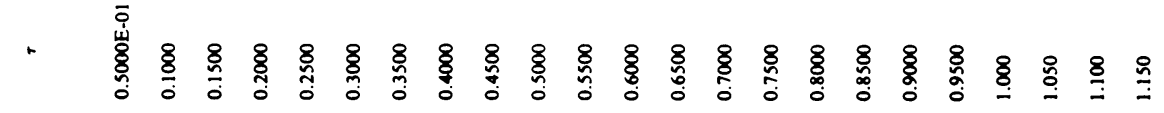

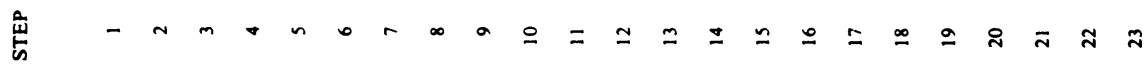
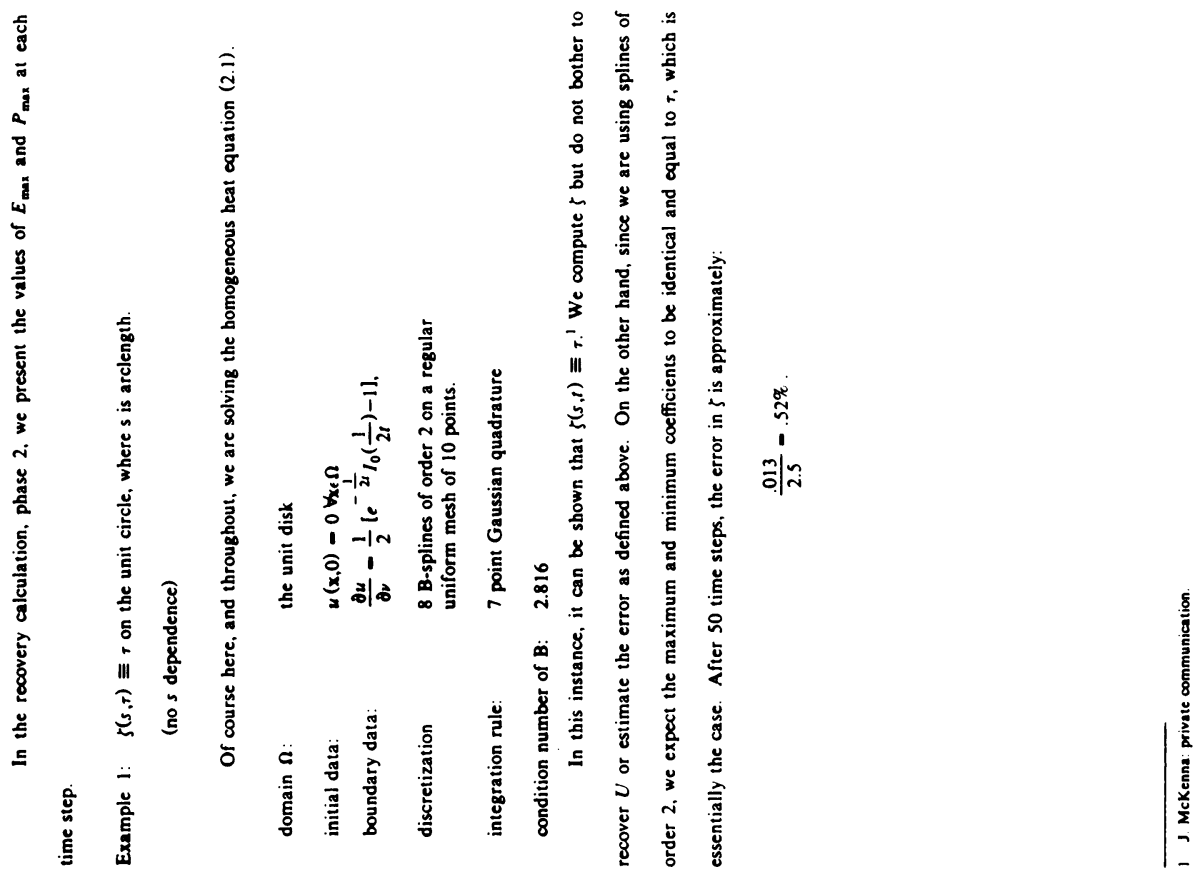


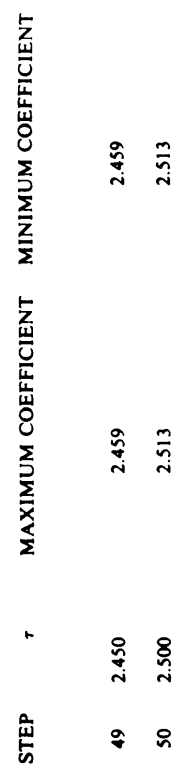

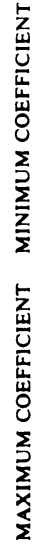

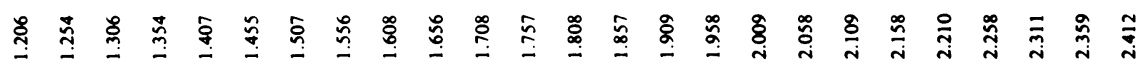

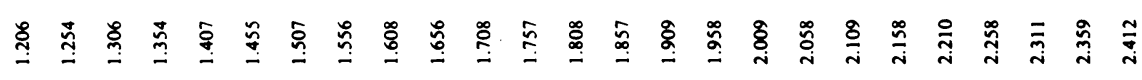

- \&

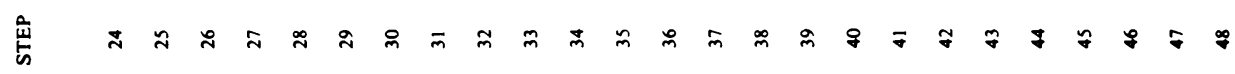



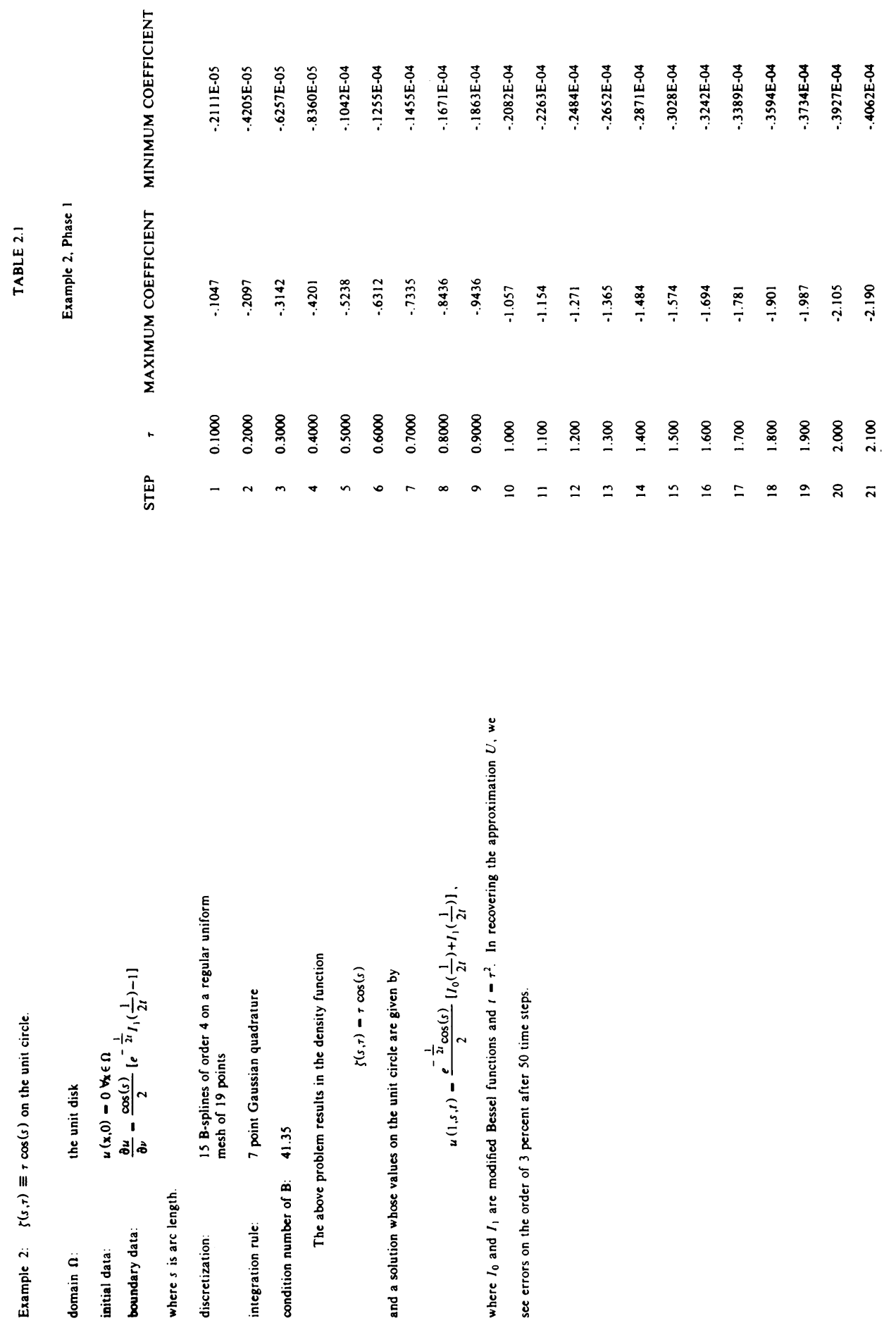

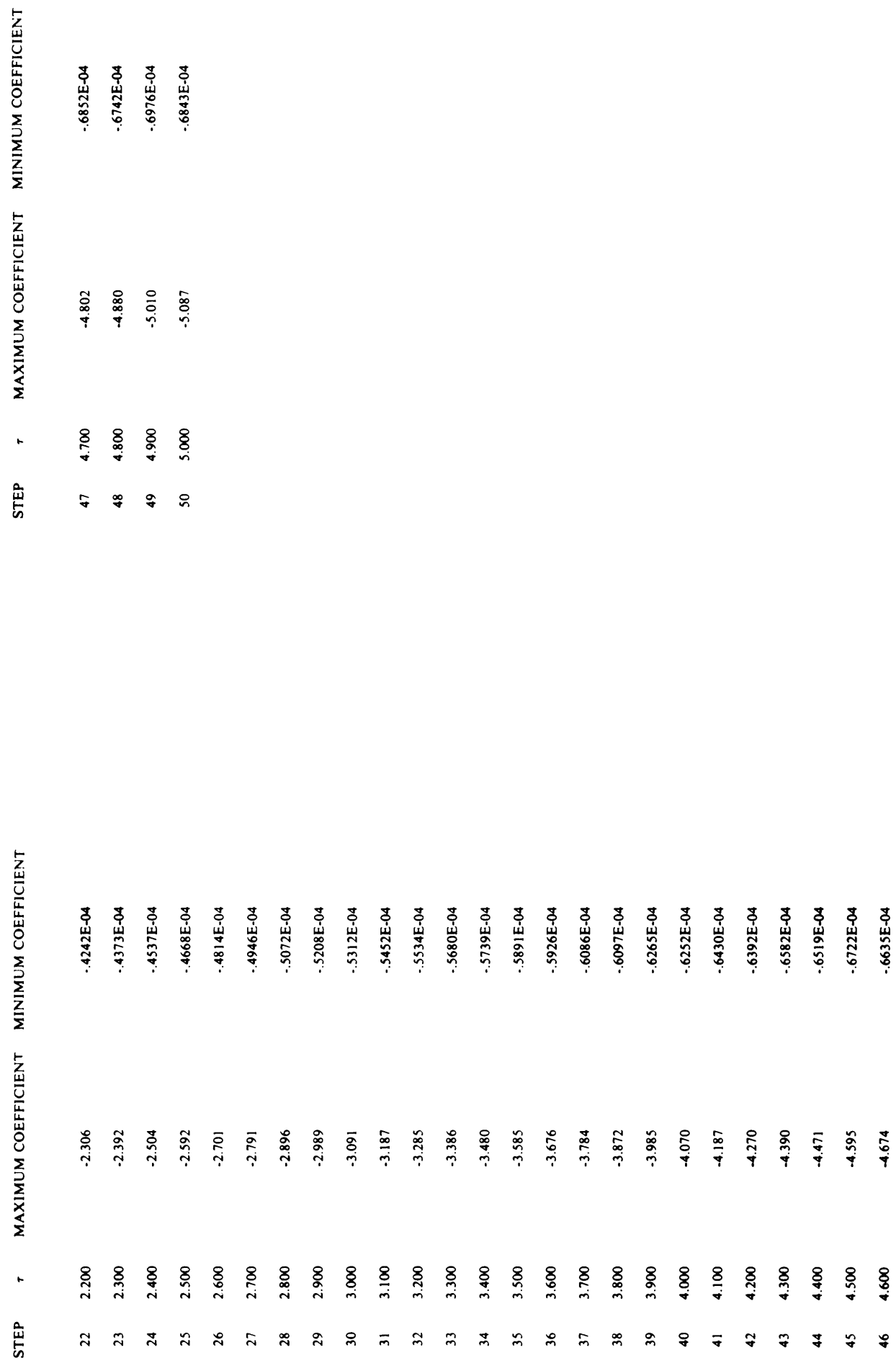


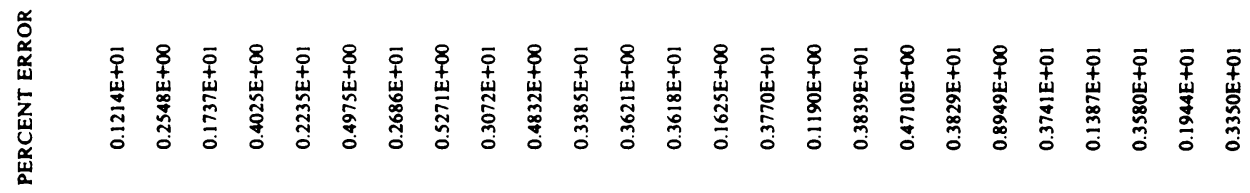

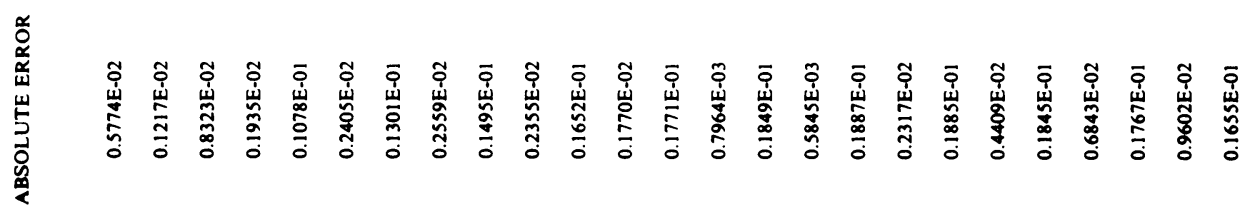

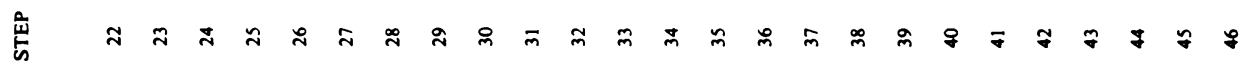

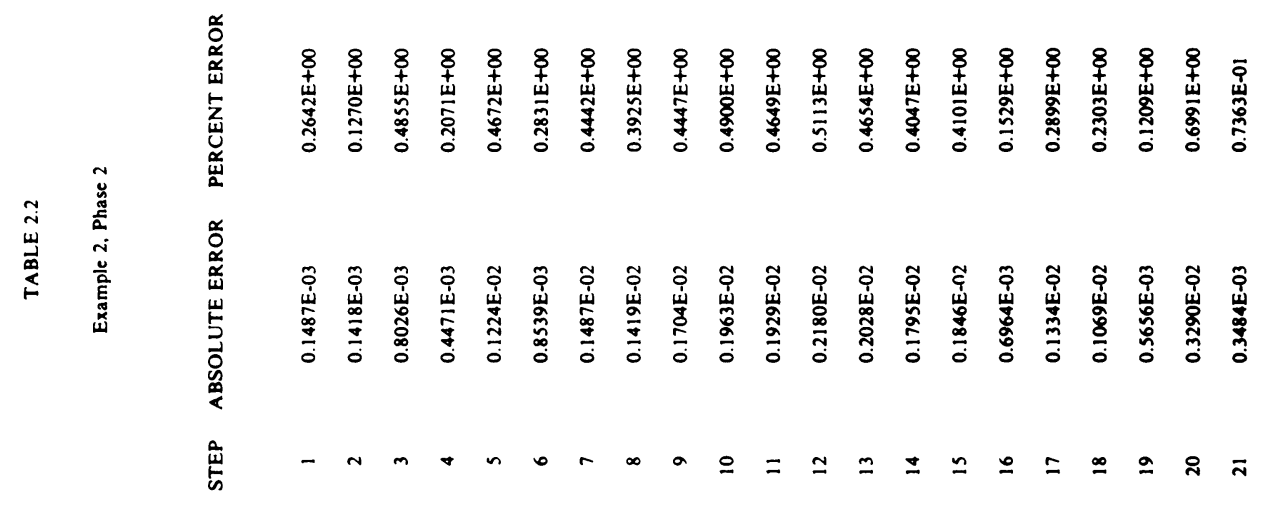



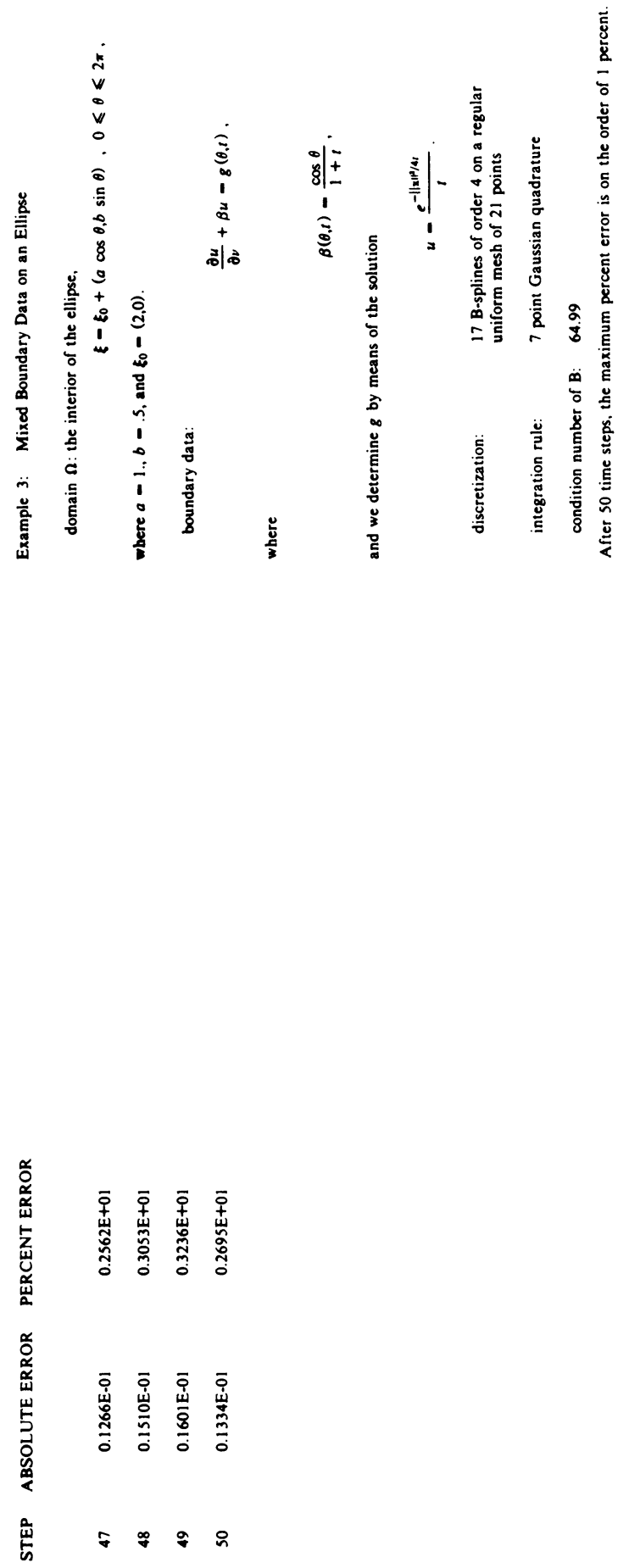


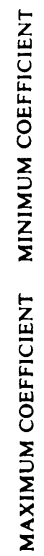

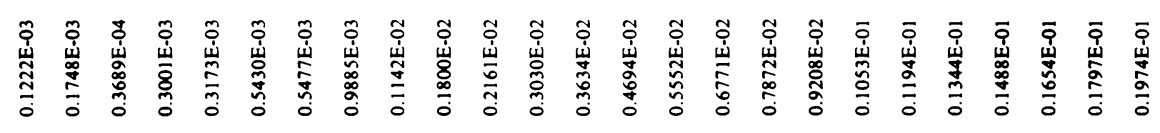

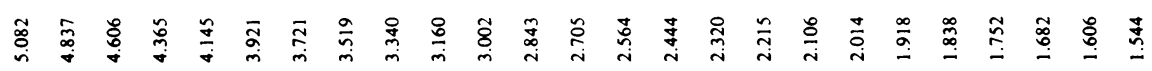

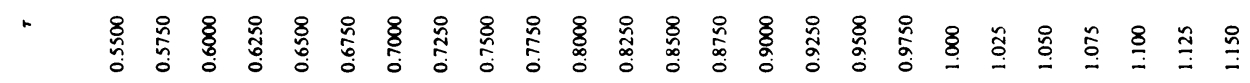

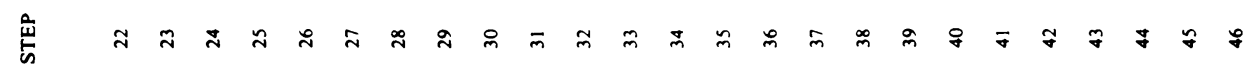

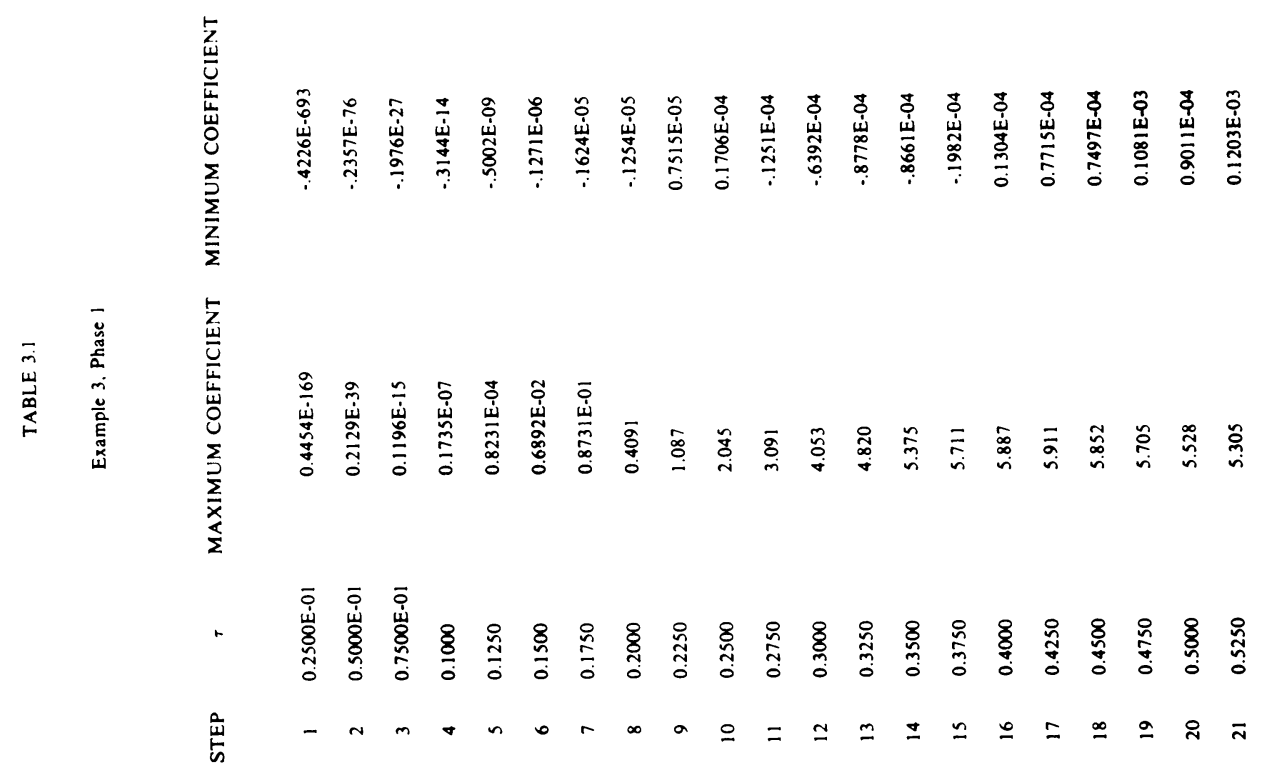



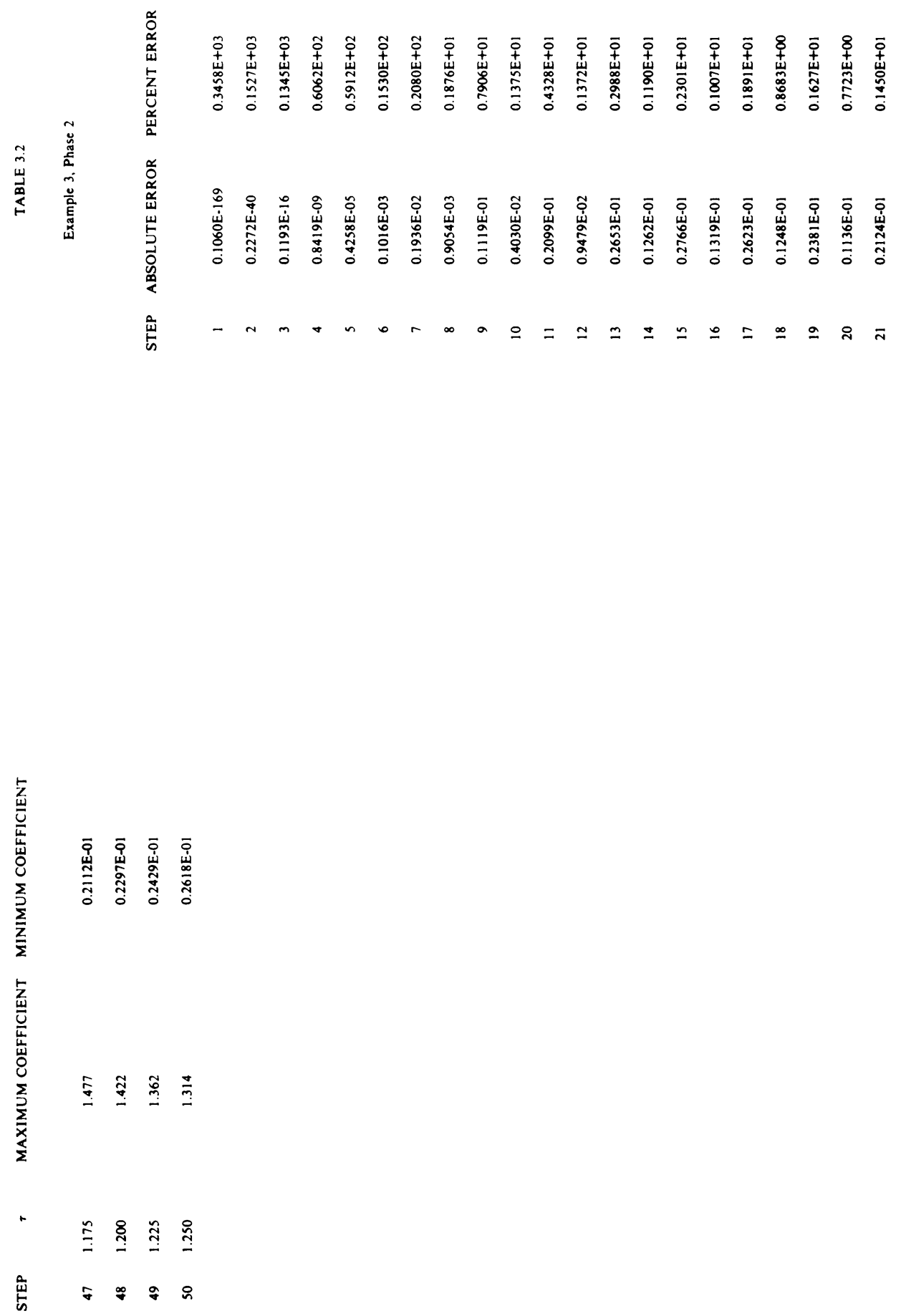

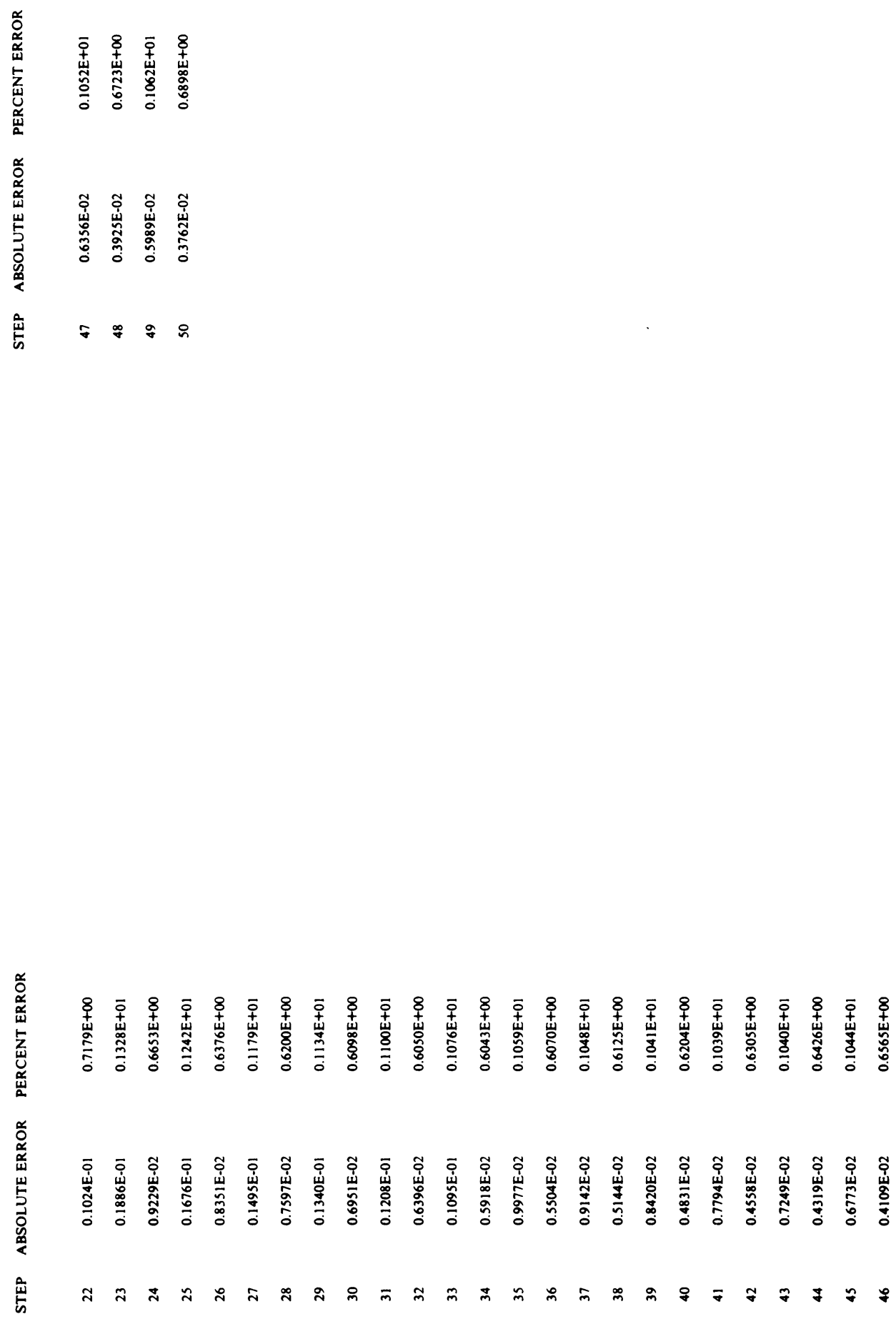

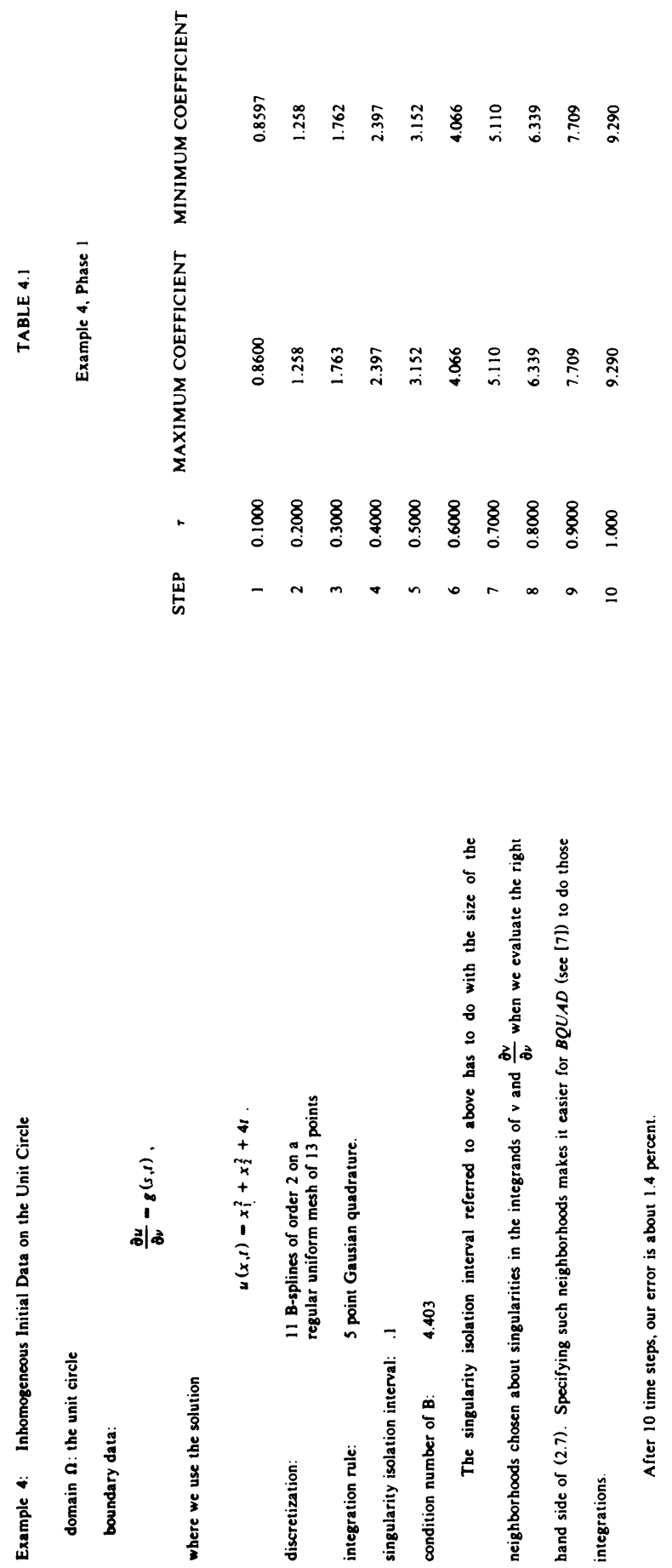

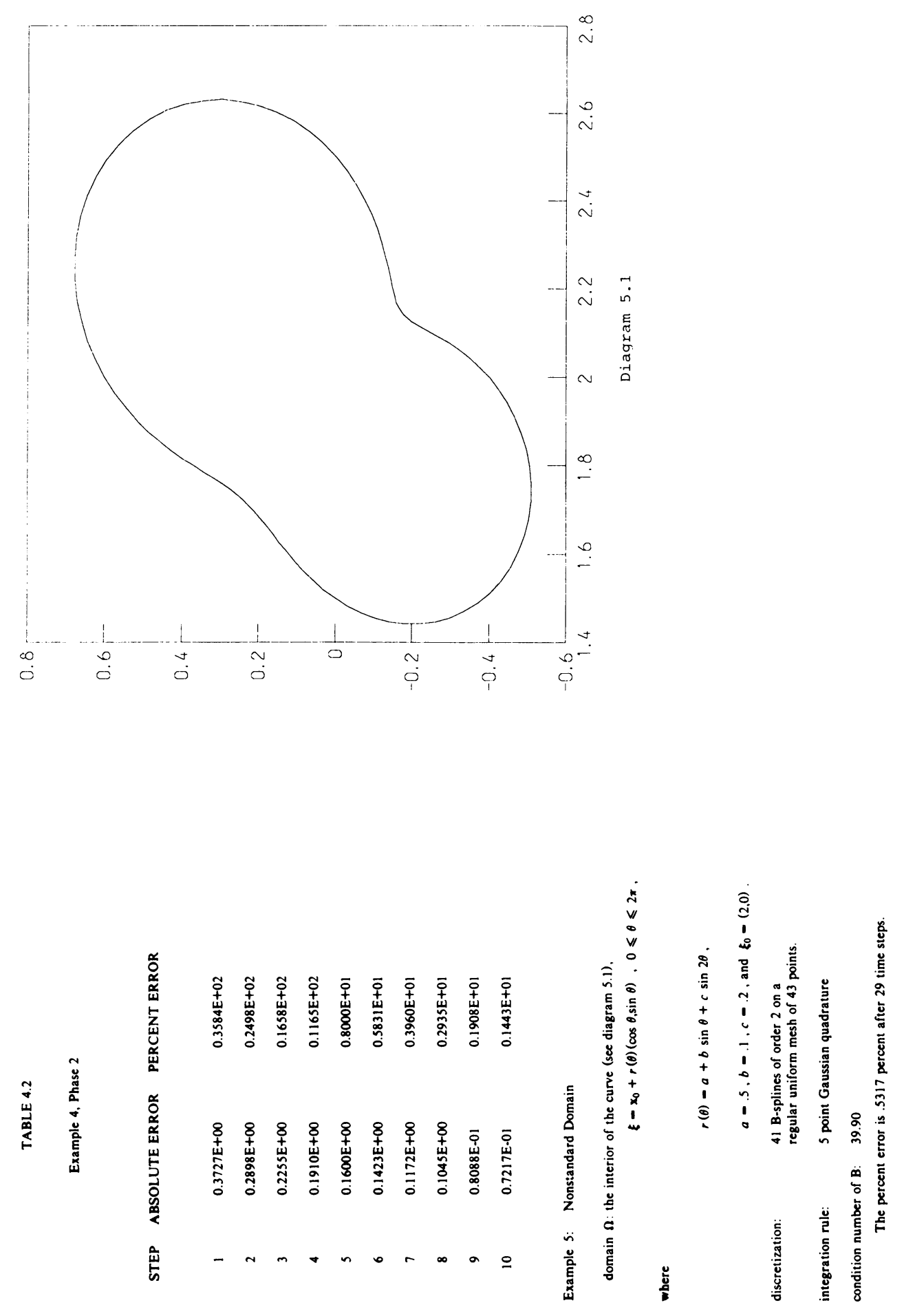

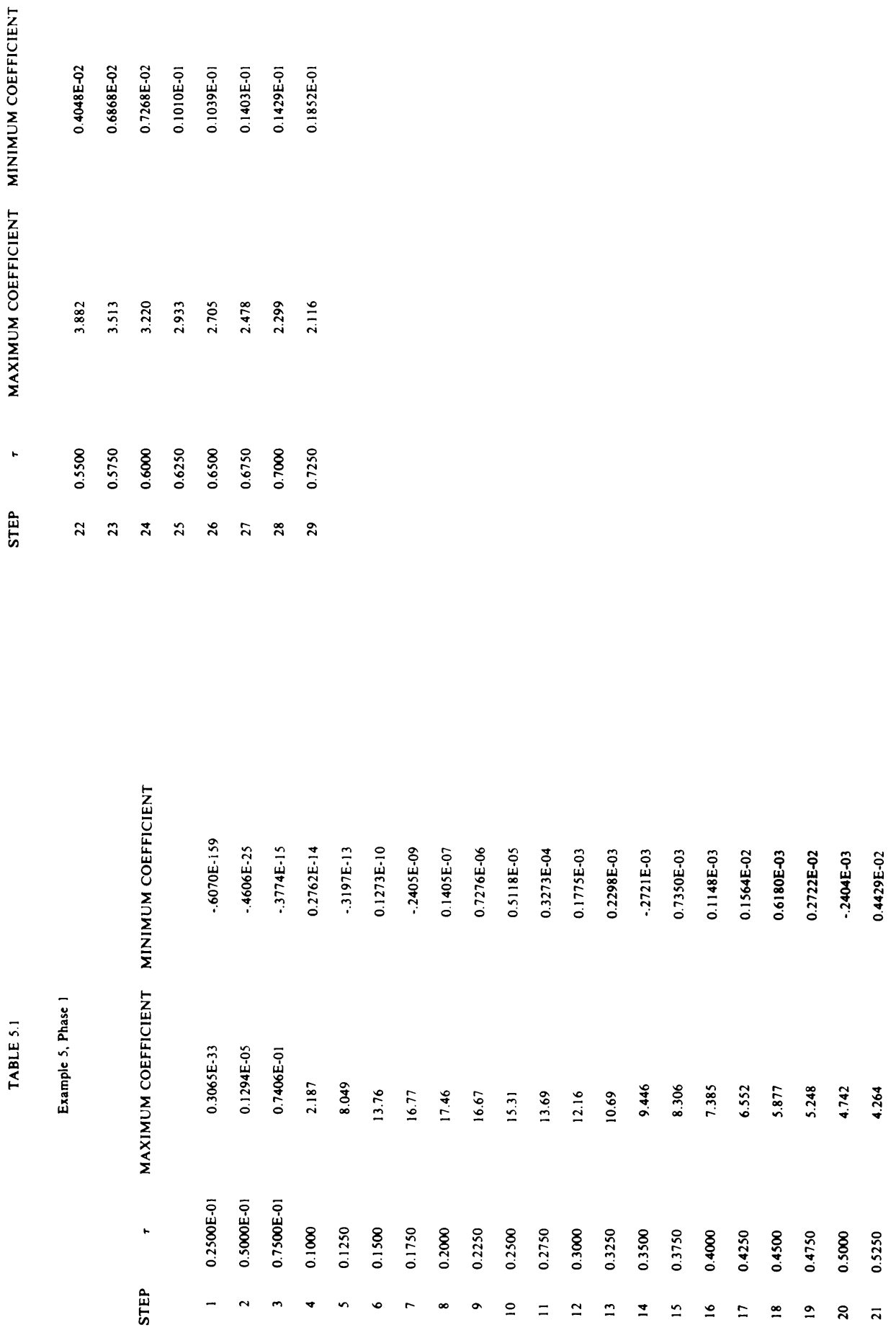

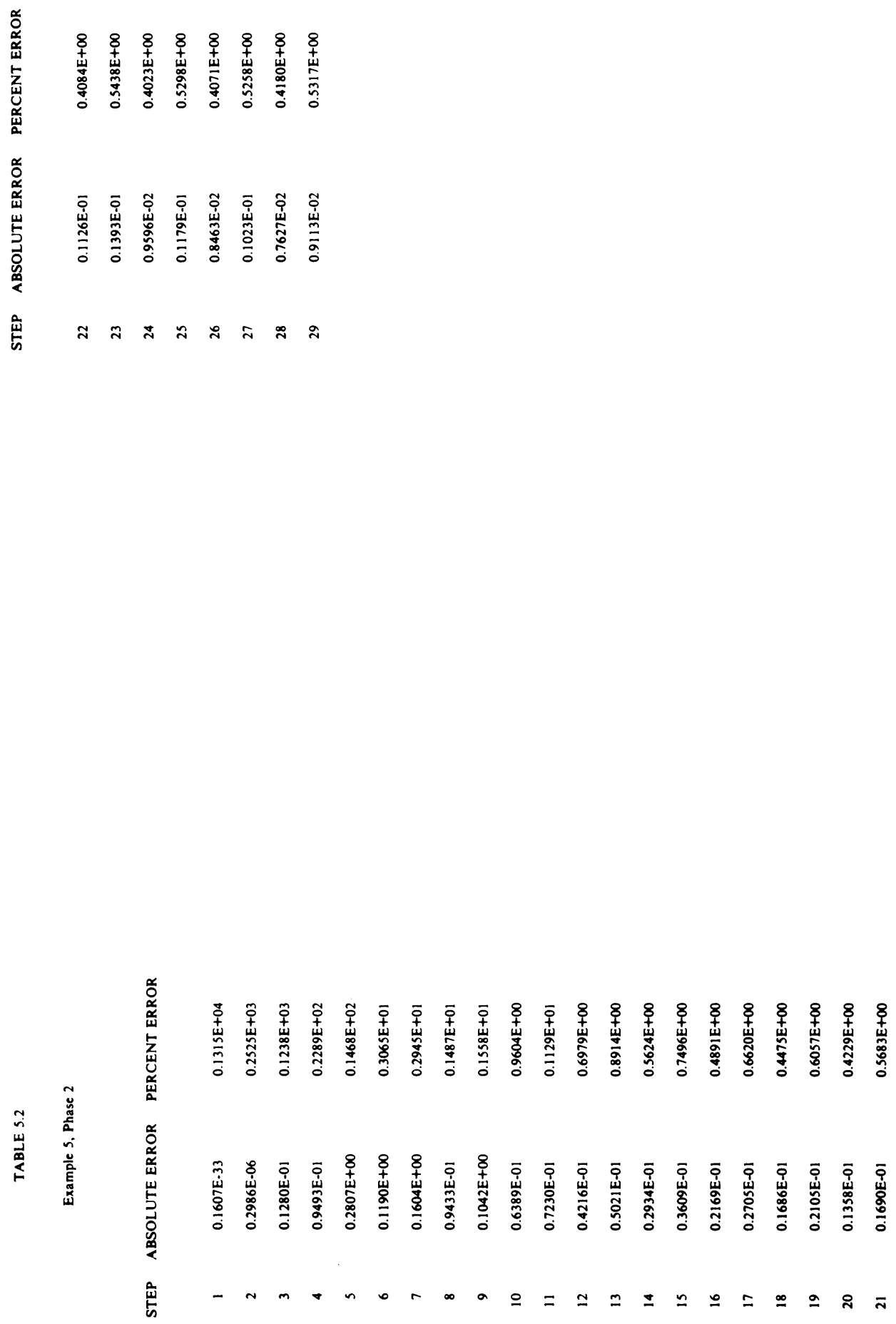